\title{
Cardiotoxicity of Non Cardiovascular Drugs: A Mechanistic Point of View
}

\author{
RASHIDT $^{\mathrm{a}}$, HASAN I ${ }^{\mathrm{b}}$
}

\begin{abstract}
Cardiotoxicity has evolved as an essential component of various adverse drug reactions and an important aspect in drug development and withdrawal. A triphasic interaction of the drug, its mechanism of action on the heart and various factors influencing toxicity is the basis of various cardiotoxicities. A comprehensive knowledge of the various mechanisms of cardiotoxicities and classes of drug causing it would promote appropriate drug selection, simultaneous
\end{abstract}

\section{Introduction}

Drugs are any chemical substances, simple or complex used in the prevention, diagnosis and treatment of diseases or abnormal conditions ${ }^{1}$. An adverse drug reaction (ADR) is a noxious and unintended response to a drug and occurs at normal doses when used for prophylaxis, diagnosis, therapy or modification of physiological function ${ }^{2}$. In some countries the ADR related costs for hospitalizations, surgery, death and loss of productivity even exceeds the cost of medications and has doubled in the last decade ${ }^{3}$. The adverse effects may be immunological like hypersensitivity reactions or drug allergies or it may be non immunological which encompasses the predictable drug toxicities like cardiotoxicities, hepatotoxicities, nephrotoxicities, CNS effects, drug interactions, drug overdose and the unpredictable idiosyncratic reactions ${ }^{4}$. According to a study in the US, the cardiac toxicity is a leading cause for drug discontinuation at all phases of the development and post market surveillance ${ }^{5}$. Worldwide $9 \%$ and in the US about $12 \%$ of all drug withdrawals were due to cardiotoxicity $^{5}$. Previously due to more conservative nature of the treatment, lack of modern treatment and adjuvant, reduced lifespan and lack of use different modalities of treatment to achieve cure, cardiotoxicity did not pose as an issue but now due to improvement of

a. Dr. Tasnuva Rashid, MD, MPH, 5401 Rampart Street, Apt 274, Houston,Texas 77081, USA.

b. Dr. Irtiza Hasan, MD 5401 Rampart Street, Apt 274, Houston,Texas 77081, USA.

Address of Correspondence: Dr. Tasnuva Rashid, MD, MPH, 5401 Rampart Street, Apt 274, Houston, Texas 77081, USA, tanvina@gmail.com, +1 (979) 229-4296

Received: 23 August 2012 Accepted 12 October, 2012 use of cardioprotective drugs and work as a guideline for the development of new drugs and targeted therapies. A check in the drug causes of cardiotoxicities would definitely play a vital role in eliminating a significant fraction of the cardiovascular diseases and delay the rate and magnitude of the development of the impending cardiovascular disease epidemic.

(Birdem Med J 2013; 3(1): 35-43)

treatment, early diagnosis, survival targeted chemotherapeutics, combination adjuvant and new adjuvant, the cardiotoxicity is posing as a clinical challenge ${ }^{6}$. According to the National Cancer Institute, cardiotoxicity means the toxicity that affects the heart ${ }^{7}$. The Cardiac Review and Evaluation Committee which supervised the Trastuzumab trial put forward certain constellation of criteria which would indicate the chemotherapy induced cardiotoxicity in terms of one or more of 1) Cardiomyopathy, either global or sepal, 2) Heart failure, 3) Signs associated with heart failure as S3 gallop, tachycardia, edema, 4) Decline in left ventricular ejection fraction (LVEF) of at least $5 \%$ to less than 55\% with sign symptoms of heart failure or asymptomatic decrease in LVEF of at least $10 \%$ to less than $55 \%{ }^{8}$. The cardiac events associated with various clinical drugs may range from mild transient increase in blood pressure or ECG changes or to more severe conditions like arrhythmia, pericarditis, myocarditis, myocardial infarction and result in left ventricular dysfunction and congestive heart failure ${ }^{9}$.

The cardiotoxicity can occur for both cardiovascular and non cardiovascular drugs. In both cases the cardiac effects can be pharmacological or toxicological where pharmacological effects occur normally due to interaction with hemodynamic and/or electrical activities of the heart through alteration of receptors or ion channel functions, more dose-dependent adverse effects, occur after the first administration of the drug and are assessed based on the pharmacological end points ${ }^{10}$. Toxicological effects result from direct cytotoxic effect on the myocardium as a result of sustained pharmacological impact, takes weeks or months to develop and detected by histopathological endpoints ${ }^{10}$. 
Based on the time to event, the cardiac events might be acute or sub acute which occur within two weeks and characterized by arrhythmia, pericarditis, myocarditis, acute heart failure or acute coronary syndromes and detected through ECG changes ${ }^{6,8}$. The chronic and more toxic effects may be classified into early toxicity occurring within 1 year of termination of the chemotherapeutics or late toxicity occurring after one year of the termination of the drug and range between asymptomatic systolic or diastolic dysfunction to left ventricular dysfunction to severe congestive Cardiomyopathy, heart failure and even death ${ }^{6,8}$.

The vascular effects may include thromboembolism, changes in blood pressure and bleeding. However for that we do need to have a comprehensive knowledge of the various mechanisms of cardiac side effects of different drugs and also the factors influencing such. The factors promoting the development of cardiotoxicity can be drug related or individual risk factors. The drug factors might include drug overdose, narrow therapeutic window, drug interactions, inappropriate time or mode of administration, pre treatment with cardiotoxic drugs, idiosyncrasy, defective metabolism and excretion of the drug and so on ${ }^{6,8}$. The individual factors include extremes of age, female gender, BMI, smoking, race, underlying heart disease, hepatic and renal impairment, low cardiac ejection fraction, genetic factors etc ${ }^{10}$. All these modify the biological interface between drug and human body and result in toxicity. The cardiotoxicity of various chemotherapeutics might be discussed on the basis of the cardiac and vascular effects. In the following sections we would briefly discuss about the varied spectrum of cardiotoxicities of non cardiovascular drugs.

\section{Cardiac toxicities}

Direct Cardiotoxicity

The cardiac dysfunction due to direct cardiotoxicity are of two types as -type 1 cardiac dysfunction is predominantly due to oxidative stress resulting in mitochondrial dysfunction, uncoupling of oxidative phosphorylation, increased mitochondrial permeability and inhibition of the metabolic pathways and type 2 cardiac dysfunction is associated with the ErbB2 pathway $^{8}$. There is a fine line of regulation between apoptotic pathways (oxidative stress) and survival pathways (HER-ErB) and an unregulated toxic apoptotic pathway coupled with inhibition of the survival pathway may result in various stages of cardiotoxicity ${ }^{11}$. The apoptotic pathways involve both reactive oxygen species (ROS) dependent pathways and ROS independent pathways ${ }^{10,11}$. The ROS dependent pathway occurs through oxidative stress resulting from the accumulation of either reactive free radicals, iron due to inhibition of iron regulatory protein (IRP1), NO, production of $\mathrm{H} 2 \mathrm{O} 2$ by outer membrane flavoprotein, peroxynitrate produced from irreversible reaction of NO with $\mathrm{O} 2$ or redox recycling resulting in numerous superoxide radicals ${ }^{10,11}$. The ROS on one hand causes disruption of the mitochondrial bioenergetics and calcium homeostasis and on the other hand results in membrane lipid peroxidation, mitochondrial DNA damage, activation of mitogen or stress activated protein kinase (MAPK, SAPK) and uncoupling of the oxidative phosphorylation $^{12}$. The aberration in the influx of calcium and resultant increase in mitochondrial calcium level results in the increase of NO production, decreased removal of H2O2, inhibition at the level of complex1 of the electron transport chain, decreased electron flow between complex 2 and 4 and thus fueling the vicious cycle $^{11}$. When this calcium concentration reaches above a certain threshold there is an increase in mitochondrial permeability transition protein (PTP) which are regulated by Cyclophillin D and increase in the permeability of the outer membrane ${ }^{10}$. All these, results in dissipation of transmembrane potential and release of cytochrome $\mathrm{C}$ into the cytoplasm which forms a complex with the adapter protein apoptosis protease activator protein 1 (Apof-1), dATP and caspase-9 and the activated caspase 9 then in turn activates other executioner caspases and results in apoptosis ${ }^{10,11}$. The other pathways include disruption of mitochondrial bioenergetics with resultant decrease in ATP synthesis and increase in ATP hydrolysis and decreased oxidative metabolism, oxidative damage to the mitochondrial lipids with degradation of myofilaments and cytoskeleton resulting in mitochondrial swelling and apoptosis ${ }^{10}$. The ROS independent pathways include inhibition of the metabolic pathways, down regulation of the genes for cellular energy supply with resultant decrease in carnitine and the inhibitory effect of carnitine on caspases and favor apoptosis , functional and structural changes in the sarcomere due to inhibition of gene transcription for sarcoplasmic reticulum Ca2+ ATPase and cardiac calcium release channels and resultant loss and disarray of myofibrils, swelling of sarcoplasmic reticulum and degradation and disorganization and down regulation of the transcription factors like P53 needed for cell survival ${ }^{10,11}$. The ROS dependent and independent pathways ultimately lead to apoptosis which if not counteracted by the survival pathways would result in myocardial cell death. 


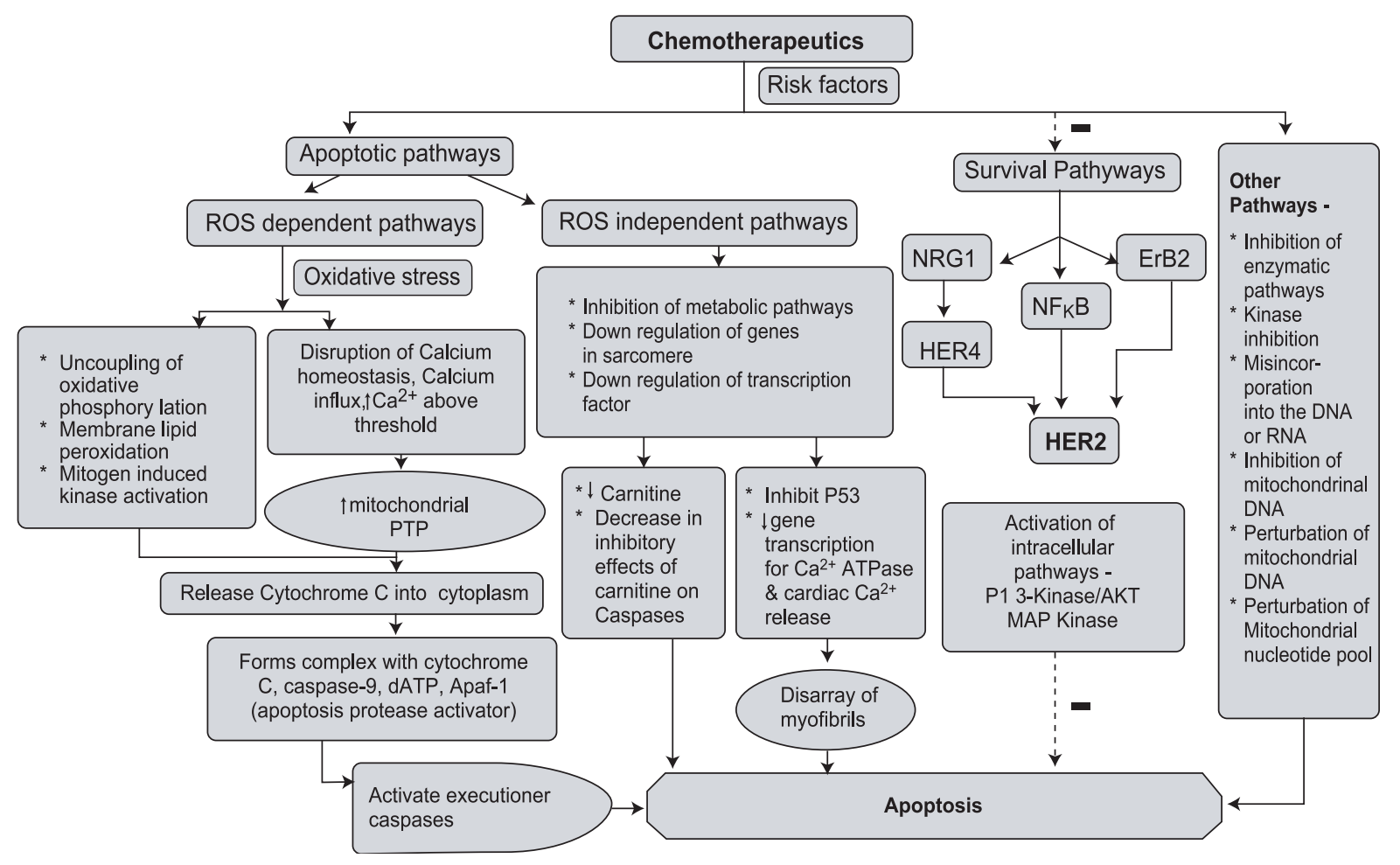

Fig 1: Mechanism of Direct cardiotoxicity

The other pathways which play a cardioprotective role are the survival pathways. The survival pathways involve HER and Neuregulin1 (NRG1) pathways and NF-KB pathway essential for restoring reperfusion and decreasing apoptosis ${ }^{13}$.The NRG1 binds to HER 4 and causes heterodimerization with HER 2 which along with ErB2 results in a complex multiple ligand cell systems and pathway interactions and subsequent activation of intracellular pathways like PI3-Kinase/AKT and MAP Kinase required for cell growth, repair and survival $^{10,11}$. All these results in survival signal. The dysregulation of the balanced coupling between the apoptotic and survival pathways may result in myocardial cell death. Various chemotherapeutics might impact at various levels of either or both the pathways with resultant cardiotoxicity. These are some of the known pathways. There might be other pathways like inhibiting various enzymatic pathways (thymidylate synthase) or getting misincorporated into the RNA or DNA, competitive kinase inhibition, depletion of mitochondrial DNA, increases mitochondrial thymidine kinase, perturbation of the endogenous mitochondrial nucleotide pool, and inhibition of mitochondrial DNA polymerase etc ${ }^{14}$.

Most of the effects of direct cardiotoxicity have been seen for Anthracyclines. It has been found that following direct toxic effect on the heart there is initially mitochondrial degeneration, interstitial fibrosis, and loss of myocardial and pericardial myocytes resulting in myocardial wall thinning, fibrosis with compensatory hypertrophy of the other unaffected cells followed by dilated and restrictive cardiomyopathy and eventually to increased left ventricular filling pressure and reduced cardiac output ${ }^{10,15,16}$.

Some major classes of drugs causing cardiotoxicity through direct mechanisms include Anthracyclines (Doxorubicin), Antimetabolites (5 FU), Alkylating agents (Cyclophosphamide), Epilithones, Cytokines (Interferon), Platinum compound (Cisplatin), Tyrosine Kinase Inhibitor (Imatinib), Monoclonal antibody (Trastuzumab), VEGF inhibitor (Sunitinib), Taxanes (Paclitaxel), Local anesthetic (Bupivacaine). 
Antiepileptic (Valproate, Phenytoin), Antidiabetics (Thiozolidinediones), Antidepressants (TCA, SSRI, MAO inhibitor), Antivirals (Non Reverse Transcriptase Inhibitor, Protease inhibitor) and Radiation ${ }^{17-27}$.

\section{Arrhythmias}

Arrhythmia is one of the pharmacological or toxicological side effects of many clinically used drugs due to interaction with various receptors and /or ion channels resulting in the impairment of action potential and rhythm disturbances of the heart. The normal action potential of the heart develops through a sequential change in the specific ionic channels like $\mathrm{Na}+, \mathrm{Ca} 2+$, channels for delayed rectifier repolarizing current (IK) made up of rapid (IKr) and slow (IKs) current, $\mathrm{Na}+-\mathrm{Ca} 2+$ exchanger and is propagated from the atria to the ventricles to maintain the normal rhythm and contractility of the heart ${ }^{10,28-31}$. Any of these ion channels might act as a target for various drugs causing arrhythmia. However most of the drugs block the delayed rectifier repolarizing (IK) channels, increase in action potential duration and results in arrhythmia ${ }^{28,}$ 29, 31. Of all the cardiac cells, the $\mathrm{M}$ cell of the ventricular muscle is particularly sensitive to the drugs that increase the action potential duration ${ }^{10}$.

Most of the arrhythmia caused by the clinical drugs is as a result of the interplay of three factors as -blockage of ionic channels, genetic predisposition and factors accelerating the drug-target interactions. The drugs preferentially either totally or partially block the rapidly activating component of the delayed rectifier current (IKr) and also sometimes the slow component (IKs) ${ }^{31}$. Blockage of the IKr is partially responsible for their proarrhythmic effects $^{10,28,29,31}$. Current is also generated by the hERG (Human Ether -a- go- go Related Gene) gene regulated channel and modulated by one of its ancillary subunit, MiRP1. The hERG current (IhERG) similar to IKr is involved in regulating the repolarization phase of the action potential (AP) from plateau voltages and in turn controls the AP duration and QT interval(10). Any drug which inhibits or blocks the current flow through the IhERG/IKr actually leads to delayed repolarization, increased QT and increased propensity to Torsades de Pointes $(\mathrm{TdP})^{10,31}$. Also, the prolongation of repolarization activates an inward depolarization current known as early after depolarization which promotes the trigger for arrhythmia. Beside potassium channel blockade, there are blockade of other channels like sodium or calcium channels which are beyond the scope of this report. The second factor involved is the genetic predisposition. The genetic mutation of the hERG or MiRP1, KCNQ1, KCNH2, KCNE1, KCNE2, SCN5A, SCN4B (associated with congenital long QT syndromes), variability of genes coding the protein which act as a target molecule for the drugs in IKr channel, mutations increasing the sensitivity of the channels to blockers and genetic absence of cytochrome enzymes related to drug metabolism might increase the cardiotoxicity and result in arrhythmias including ventricular fibrillation $28,29,32,33$.

The third factor which plays a vital role in modulating the nature of the drug target interaction resulting in arrhythmia is the associated risk factors. Due to "repolarization reserve" which is based on the concept of simultaneous action of multiple ions on the repolarization and translating into the fact that the blockage of one ion channel might not result in clinically visible torsades ${ }^{10,28}$. As such the level and interplay of other risk factors might accelerate the process into clinical manifestation. The various factors includes extremes of age, female gender, racial predilections, metabolic abnormalities, electrolyte imbalance (hypokalemia mostly, hypomagnesaemia, hypocalcaemia), hepatic impairment, renal insufficiency, genetic predisposition and history of long QT syndrome ${ }^{31}$. The various drug related factors include drug overdose, multiple drug use, drug interaction, defective metabolism and excretion, concomitant use of QT prolonging drug, enzyme induction, enzyme inhibition and use of drugs with narrow therapeutic window ${ }^{10,28,31,34}$. The presence of organic heart diseases like ischemic heart disease, congestive heart failure, dilated Cardiomyopathy, hypertrophic Cardiomyopathy, myocarditis, bradycardia, atrioventricular block, sinoatrial block, recent conversion from atrial fibrillation and so on ${ }^{31}$. All these factors might reduce the net repolarizing current and in turn reduce the repolarization reserve and cause various symptoms and manifestations. 


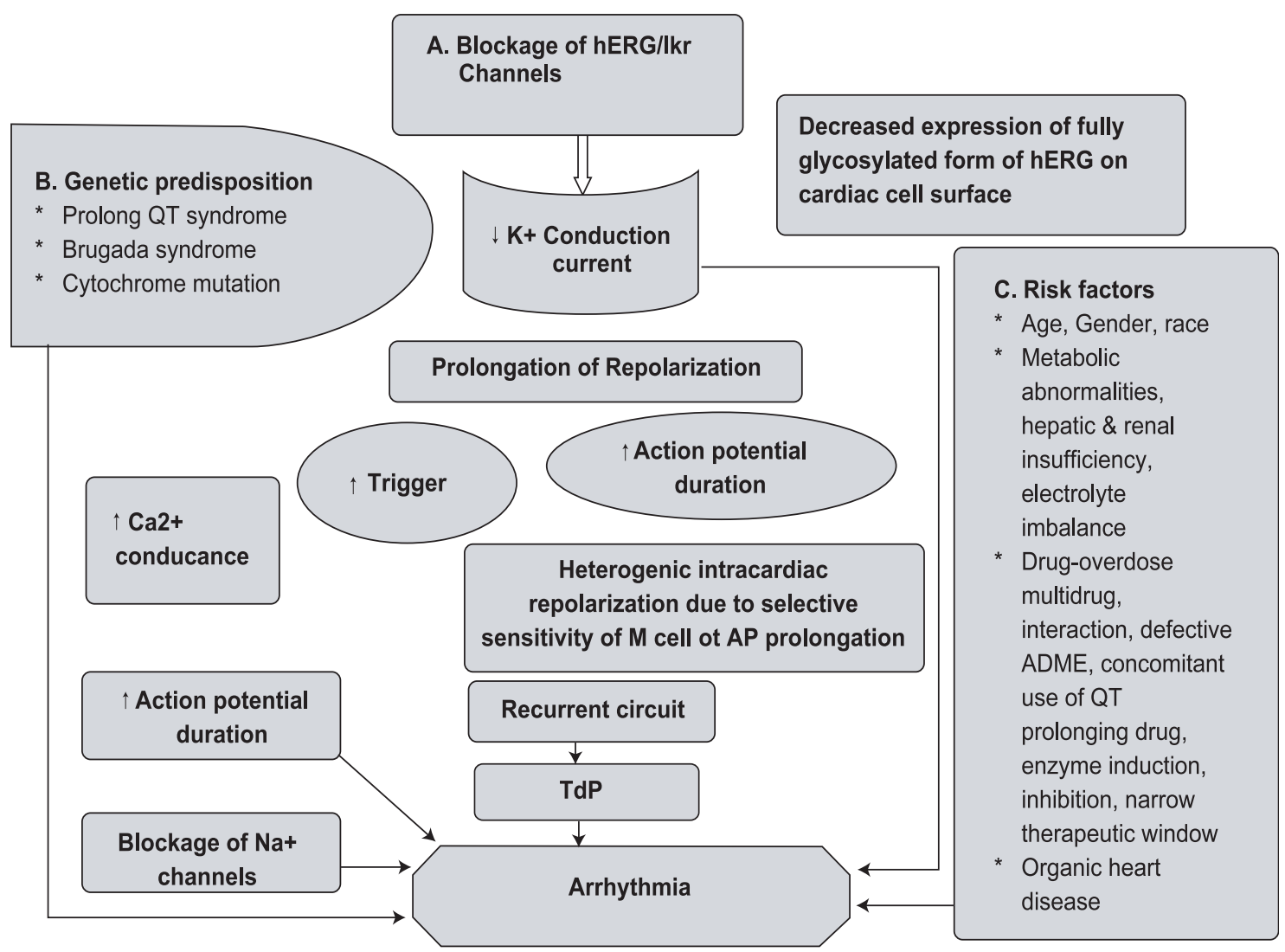

Fig.-2: Mechanism of Arrhythmia

Some of the major classes of non cardiovascular drugs having the propensity to cause arrhythmia include Antihistamines (Diphenhydramine, Chlorpheniramine, Loratidine), Antipsychotics (Phenothizines, Butyrophenones, Risperidone, Clozapine), Antidepressants (TCA, Bupropion, Venlafaxine, Trazodone),

Lithium, Antibacterial (Erythromycin, Clarithromycin, Sparfloxacin, Moxifloxacin, Gratifloxacin, Levofloxacin, Ciprofloxacin), Antifungals (Ketoconazole, Fluconazole, Itrconazole), Antivirals (Amantidine), Antiprotozoal (Quinine, Chloroquine, Pentamidine, Sodium Antimony), Droperidol, Famotidine, Anthracyclines, Monoclonal antibody (Rituximab), Tyrosine Kinase Inhibitor (Nitotinib), Antimetabolites (5FU), Histone Deacetylase, Taxanes (Paclitaxel), Methotrexate, Cytokines (Interferon) and Tacrolimus ${ }^{10,35}$.

\section{Contractile Dysfunction}

Contractile dysfunction is one of the drug related cardiotoxicities which might lead to Cardiomyopathy ${ }^{36,37}$.
The mechanisms might include either cardiac dysfunction due to cell death via the mitochondrial electron chain inhibition pathway or reversible dysfunction due to temporary dysfunction of the myocytes contractile element resulting in systolic dysfunction. There might be endothelial myocytes coupling disorder ${ }^{36}$. The anticancer drugs and steroid are the common causes of Cardiomyopathy ${ }^{10}$. The common non cardiovascular drugs causing contractile dysfunction includes Anthracyclines, Trastuzumab, Sunitinib, Tyrosine Kinase inhibitors, alphaInterferon, Cyclophosphamide, steroids, testosterone etc ${ }^{10,36}$. There might also be diastolic dysfunction resulting from hypertrophy, fibrosis, infiltrative diseases, storage diseases and pericardial constriction as a result of ischemia, cellular calcium overload, ATP depletion and ultimately result in congestive heart failure ${ }^{37}$.

\section{Vascular toxicities}

The vascular effects of the clinical drugs can be grossly related to clotting system and variation of vascular resistance. 


\section{Clotting system}

The normal anti thrombotic nature of the circulatory system is maintained through a balance between the vascular endothelial function, blood flow and blood constituents (Virchow's triad) and any abnormality in any or all of these three components of Virchow's triad may lead to thrombosis and thromboembolism both in the arterial and venous system ${ }^{38}$. The altered blood flow might occur due to either vasoconstriction or vasodilatation and influence thrombogenic state. Some of the drugs might potentiate the effects of the vasoconstrictors or increase the contractility of the blood vessels or reduce $\mathrm{NO}$ and cause vasospasm and some cause vasodilatation ${ }^{38}$. An intact endothelium is an essential component to prevent the formation of thrombosis. Impairment of endothelial function is associated with oxidative stress, increased release of Von Willibrands factor, endothelin, tissue factor, tissue plasminogen activator along with expression of various adhesion and recognition molecules like E -selectin, release of inflammatory mediators like TNF, IL1 and metabolically active structures as thrombomodulin and binding sites for the clotting factors ${ }^{39}$. These results in abnormal vascular compliance and prothrombotic status $^{39,40}$. The angiogenesis factors like VEGF (Vascular Endothelial Growth Factor), PDGF (Platelet derived growth factor) inhibition may lead to decreased endothelial integrity, cause defects in the endothelium exposing the thrombogenic phospholipids and decrease in NO and PGI2 which will all predispose to thromboembolic events ${ }^{39,40}$. The proteolysis of the endothelial cell and matrix junction may also expose the thrombogenic basement membrane. The increased secretion of the prothrombotic factors, increase in clotting factors, decrease in antithrombotic factors, decrease in antithrombotic factors like protein $\mathrm{C}$, protein $\mathrm{S}$, increased level of serotonin, antiphospholipid antibody, and decrease in the clearance of the clotting factors may all lead to the thrombogenic environment ${ }^{38}$, 39. A selective inhibition of COX2 enzyme results in removal of antioxidant protection of the endothelium and decrease in PG I2, a potent vasodilator and inhibitor of platelet aggregation which in turn results in the promotion of atherogenesis and thrombosis along with uninhibited action of thromboxane A2 and hence may result as a possible trigger for cardiovascular toxicity(41). Besides, inhibition of NO, oxygenation of endocannabinoid 2-arachidonoyl glycerol to its prostaglandin derivatives would inhibit PPAR _ and in turn stimulate prothrombotic factors and tissue factors $^{10,41}$. Thus an endothelial dysfunction, shift of the balance of coagulation system towards

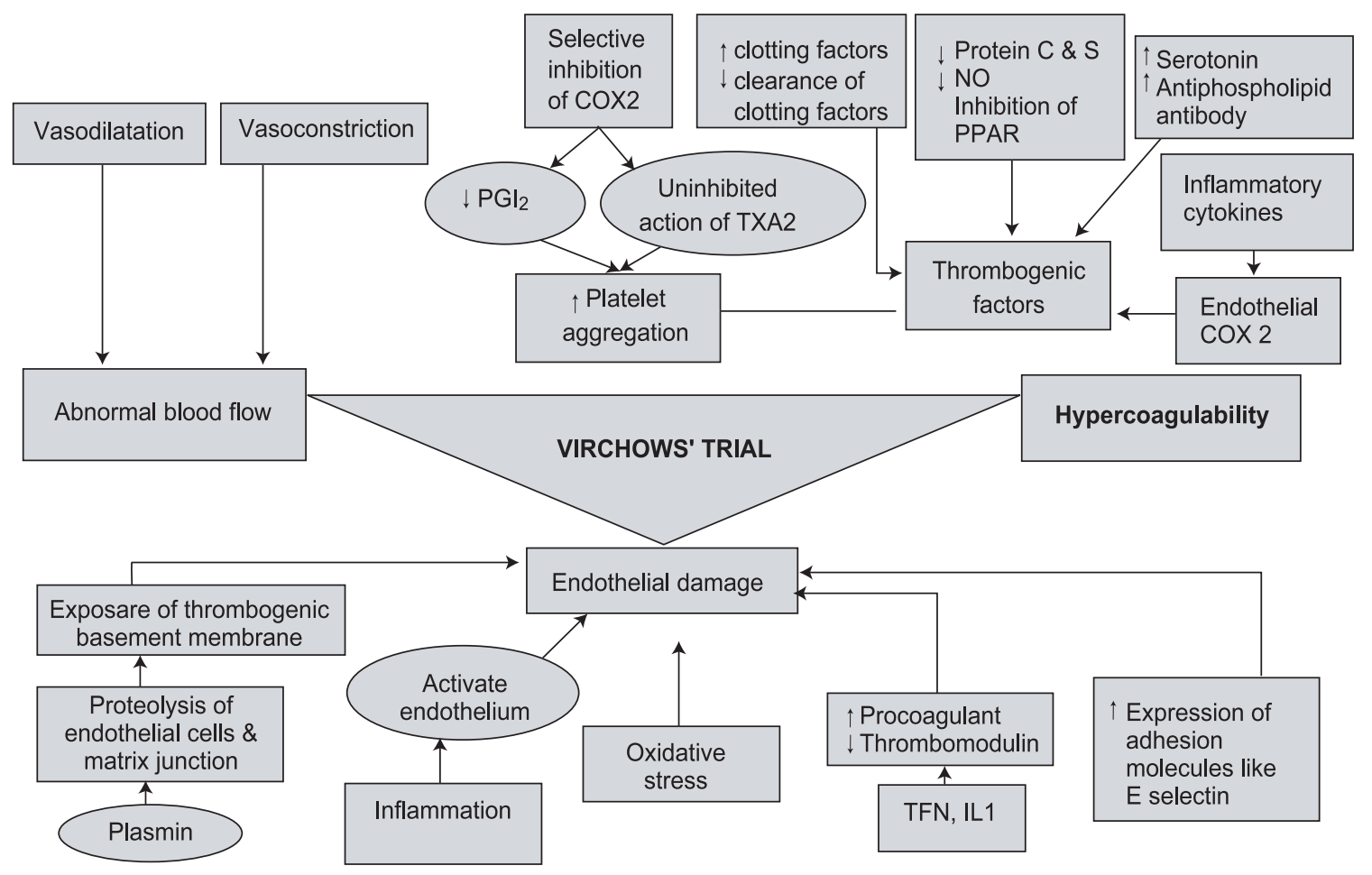

Fig 3: Mechanisms of cardiotoxicity involving clotting system 
procoagulant, together with stasis or abnormal blood flow results in thrombosis and thromboembolism in both cardiac and peripheral vessels.

Some drugs might cause hemorrhage through causing thrombocytopenia or increasing the clearance of clotting factors. These include quinine, quinidine, cotrimoxazole, rifampin, NSAIDs, Aspirin, Penicillin, Cephalosporin and steroids. Hemorrhage is also a common sideffect of the anticoagulants ${ }^{39}$.

Some of the major classes of drugs affecting the clotting system includes, Alkylating agents (Cyclophosphamide), Methotrexate, Topoisomerase inhibitors (Etoposide), Microtubule inhibitors (Paclitaxel, Vincristine, Vinblastine), Antitumor antibody (Mitomycin, Bleomycin), Platinum compounds (Cisplatin), Hormone Replacement Therapy (Tamoxifen, Raloxifen), Rapamycin, Monoclonal antibody (Bevacizumab), Vasopressin, Nicotinic acid, Estrogen, Cyclosporine, TNF agents (Etanarcept), Steroid, Angiotensin II, Granulocyte Monocyte Colony Stimulating Factor (GMCSF), Tissue Plasminogen Activator (TPA), Heparin, Anthracyclines, Tyrosine Kinase Inhibitors, Selective Serotonin Reuptake Inhibitor (SSRI), Antipsychotics (Chlorpromazine, Fluphenazine, Haloperidol), Oral Contraceptive Pills, NSAIDs (Ibuprofen, Indomethacin, Diclofenac, Coxibs) and Immune Modulating drugs (Linelidomide, Pomalidomide) $)^{38-44}$.

\section{Vascular Resistance}

Chemotherapeutic might increase the vascular resistance through a decrease in the angiogenic factors like vascular endothelial growth factor (VEGF), platelet derived growth factor (PDGF), decrease in NO synthase activity and activation of plasminogen activator inhibitor 1 , increase in calcium sensitivity of the myofilaments and increase release of serotonin and dopamine which all results in vasospasm and ultimately hypertension ${ }^{45}$. Some chemotherapeutics like Rituximab and Propofol causes hypotension through peripheral vasodilatation and reduced myocardial contractility. Some non cardiovascular drugs which cause hypertension includes, Epinephrine, Erythropoietin, Steroid, NSAIDs (Aspirin, Ibuprofen, Naproxen), Antidepressants (TCA, Venlafaxine, Bupropion), HRT, OCP, Sumatriptan, Ergotamine, Monoclonal Antibody (Bevacizumab), Interferon, Cyclosporine, 5FU, Cisplatin, Sunitinib and
Radio immunotherapy (Ibritumomabtiuxetan, Gemtuzumabozogamicin $)^{45-55}$.

\section{Teratogenic effect on cardiac development}

There are many drugs that have a teratogenic effect on the heart but among them the most significant ones are lithium, isotretinoin, folate antagonists, NSAIDs, 5HT blockers and benzodiazepines ${ }^{56}$. Lithium is a drug used in bipolar disorder which if taken during pregnancy might cause Ebsteins' Anomaly ${ }^{56}$. According to various studies it has been found that Lithium is a weak Teratogen. The mechanism with this teratogenic effect is linked to interruption of apoptosis of the right side of the heart and corresponding restriction in the growth of tricuspid valve and chordae tendinae ${ }^{57}$. Folic acid antagonists like Phenobarbital, Phenytoin, Carbamazepine, Trimethoprim, methotrexate etc if taken during the 2nd or 3rd trimester is associated with cardiovascular defect ${ }^{58}$. Altering the level of folic acid or homocysteine is associated with abnormalities with cardiac neural crest cell migration, differentiation and cell cycle progression ${ }^{58}$. Proper level of retinoic acid is required for the neural crest cell development ${ }^{58}$. Embryonic retinoic acid is synthesized by retinal dehydrogenases which if inhibited might set the retinoid homeostasis in disarray ${ }^{58}$. Isotretinoin used in the treatment of acne, when to given to pregnant patient on one hand blocks the retinal dehydrogenase and on the other hand bind with retinoic acid receptors (RARs) and retinoid X receptors (RXRs), which are transcription factors and affects the genes involved neural crest development and result in cardiac malformation ${ }^{58}$. NSAID if used specially in the first trimester is associated with cardiac septal defect ${ }^{58}$. 5 HT receptor blockers like Quetiapine, Risperidone, and Granisetron are associated with defects in cardiac morphogenesis. An association has also been found between Benzodiazepine and cardiac malformation ${ }^{60}$. These are some of the embryonic cardiotoxicity of clinical drugs.

\section{Conclusion}

The cardiotoxicity of non cardiovascular drugs is one of the preventable risk factors for the development of the cardiovascular disease and an important target for intervention. There is an increasing need to appreciate the different modes of cardiotoxicity induced by drugs, to stress on the risk factors, cardiovascular monitoring, identification of at risk population, determination of the pros and cons of combination therapy with overlapping 
cardiotoxicity and dose adjustment. The clinical use of cardioprotectants coupled with early cardiac biomarkers would be an efficient step towards the solution. There is a need for newer drug developments keeping in mind the mechanisms and their complex interaction with the variability of the risk factors at the level of the individual, at drug delivery and environment of the interaction. A well visioned step towards controlling cardiotoxicity would definitely help to reduce a portion of the cardiac disease burden.

\section{References}

1. Farlex. The free dictionary. 2012; Available from: <http:/ /medicaldictionary. thefreedictionary.com/drug>

2. VHA. Adverse Drug Events, Adverse Drug Reactions and Medication Errors. 2006; Available from: http://www. pbm.va.gov/vamedsafe/Adverse\%20Drug\%20 Reaction. pdf

3. WHO. Medicines: safety of medicines - adverse drug reactions. 2008; Available from: http://www.who.int/ mediacentre/factsheets/fs293/en/index.html.

4. AAFP. Adverse Drug Reactions: Types and Treatment Options. 2003; Available from: http://www.aafp.org/afp/ 2003/1101/p1781.html.

5. Piccini JP, Whellan DJ, Berridge BR, Finkle JK, Pettit $\mathrm{SD}$, Stockbridge $\mathrm{N}$, et al. Current challenges in the evaluation of cardiac safety during drug development: Translational medicine meets the Critical Path Initiative. American heart journal2009;158(3):317-26.

6. Albini A, Pennesi G, Donatelli F, Cammarota R, De Flora S, Noonan DM. Cardiotoxicity of anticancer drugs: the need for cardio-oncology and cardio-oncological prevention. Journal of the National Cancer Institute;102(1):14-25.

7. NCI. Dictionary of Cancer Terms. 2012; Available from: http://www.cancer.gov/dictionary?cdrid=44004.

8. Fiuza M, Magalhã£es A. Trastuzumab and Cardiotoxicity.

9. Gharib M, Burnett A. Chemotherapy-induced cardiotoxicity: current practice and prospects of prophylaxis. European Journal of Heart Failure 2002; 4(3):235-42.

10. GiorgioMinotti. Cardiotoxicity of Non-Cardiovascular drugs 2010. Available from: http://books.google.com/ books/about/Cardiotoxicity_of_Non_Cardiovascular_ Dru.html?id=h8kNNv3C3 VwC.

11. Montaigne D, Hurt C, Neviere R. Mitochondria Death/ Survival Signaling Pathways in Cardiotoxicity Induced by Anthracyclines and Anticancer-Targeted Therapies. Biochemistry Research International;2012.

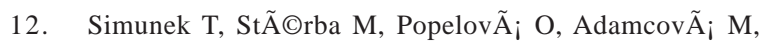
Hrdina R, Gersl V. Anthracycline-induced cardiotoxicity: overview of studies examining the roles of oxidative stress and free cellular iron. Pharmacol Rep2009;61(1):154-71.

13. Crone SA, Zhao YY, Fan L, Gu Y, Minamisawa S, Liu Y, et al. ErbB2 is essential in the prevention of dilated cardiomyopathy. Nature medicine 2002; 8(5): 459-65.

14. Force T, Kolaja KL. Cardiotoxicity of kinase inhibitors: the prediction and translation of preclinical models to clinical outcomes. Nature Reviews Drug Discovery; 10(2):111-26.

15. Puma N, Ruggiero A, Ridola V, Maurizi P, Lazzareschi I, Attinã• G, et al. Anthracyclinerelated cardiotoxicity: risk factors and therapeutic options in childhood cancers. Signa Vitae2008; 3(1): 30-4.

16. WoltersKluwer. Cardiotoxicity of anthracycline-like chemotherapy agents 2012; Available from: http:// www.uptodate.com/contents/cardiotoxicity-ofanthracycline-likechemotherapy-agents.

17. Su C, Kung C, Wang Y, Lu C. Life-threatening cardiotoxicity due to chronic oral phenytoin overdose. Neurology India 2009; 57(2): 200.

18. Minotti G, Menna P, Salvatorelli E, Cairo G, Gianni L. Anthracyclines: molecular advances and pharmacologic developments in antitumor activity and cardiotoxicity. Pharmacological reviews2004;56(2):185-229.

19. Alter P, Herzum M, Soufi M, Schaefer J, Maisch B. Cardiotoxicity of 5-fluorouracil. Cardiovascular \&\# 38; Hematological Agents in Medicinal Chemistry (Formerly Current Medicinal Chemistry- Cardiovascular \&\# 38; Hematological Agents)2006;4(1):1-5.

20. Becker K, Erckenbrecht JF, Haaussinger D, Frieling T. Cardiotoxicity of the antiproliferative compound fluorouracil. Drugs1999;57(4):475-84.

21. Abdel-Nabi IM, Omran MA. Cyclophosphamide-induced early cardiotoxicity: Comparison of ECG changes and disorders in adult and senile rats. Egyptian Journal of Biology2004;1(1):1-15.

22. Sonnenblick M, Rosin A. Cardiotoxicity of interferon. A review of 44 cases. Chest1991;99(3):557-61.

23. Mather LE, Chang DHT. Cardiotoxicity with modern local anaesthetics: is there a safer choice? Drugs 2001; 61(3):333-42.

24. Dippenaar J. Local anaesthetic toxicity. Southern African Journal of Anaesthesia and Analgesia;13(3):23-8.

25. Aljada A, Shah KA, Mousa SA. Peroxisome proliferatoractivated receptor agonists: do they increase cardiovascular risk? PPAR research2009;2009.

26. eMedExpert. Comparing Selective Serotonin Reuptake Inhibitors (SSRIs) to Tricyclic Antidepressants (TCAs). 2012. 
27. Munger MA, Effron BA. Amoxapine cardiotoxicity. Annals of emergency medicine1988;17(3):274-8.

28. Roden DM. Pharmacogenetics and drug-induced arrhythmias. Cardiovascular research2001;50(2):224-31.

29. Liu K, Yang T, Viswanathan PC, Roden DM. New Mechanism Contributing to Drug-Induced Arrhythmia. Circulation2005;112(21):3239-46.

30. Barnes BJ, Hollands JM. Drug-induced arrhythmias. Critical care medicine;38:S188.

31. Yap YG, Camm AJ. Drug induced QT prolongation and torsades de pointes. Heart2003;89(11):1363-72.

32. Crotti L, Celano G, Dagradi F, Schwartz PJ. Congenital long QT syndrome. Orphanet J Rare Dis2008;3:18.

33. NIH. Brugada Syndrome. 2006; Available from: http:// ghr.nlm.nih.gov/condition/brugadasyndrome.

34. Olshansky S, Ault A. The fourth stage of the epidemiologic transition: the age of delayed degenerative diseases. The Milbank Quarterly1986;64(3):355-91.

35. ArizonaCert. QT Drug Lists by Risk Groups. 2012.

36. Klimas J. Drug-Induced Cardiomyopathies.

37. Apstein CS, Morgan JP. Cellular mechanisms of diastolic dysfunction. 2000.

38. Ramot Y, Nyska A. Drug-Induced Thrombosisâ•” Experimental, Clinical, and Mechanistic Considerations. Toxicologic pathology2007;35(2):208-25.

39. LaurenL.Patton. Bleeding and Clotting Disorder. Available from: http://web.squ.edu.om/med- Lib/MED_CD/E_CDs/ oral\%20medicine/docs/ch17.pdf.

40. Chong AY, Blann A, Lip G. Assessment of endothelial damage and dysfunction: observations in relation to heart failure. QJM2003;96(4):253-67.

41. Mukherjee D, Nissen SE, Topol EJ. Risk of cardiovascular events associated with selective COX-2 inhibitors. JAMA: The Journal of the American Medical Association2001;286(8):954-9.

42. Madhusudan S, Harris AL. Drug inhibition of angiogenesis. Current opinion in pharmacology2002;2(4):403-14.

43. Wu H, Huang C, Chang D. Anti-angiogenic therapeutic drugs for treatment of human cancer. J Cancer Mol2008;4(2):37-45.

44. Wolf MB, Baynes JW. The anti-cancer drug, doxorubicin, causes oxidant stress-induced endothelial dysfunction. Biochimica et Biophysica Acta (BBA)-General Subjects2006;1760(2):267-71.

45. Neusser M, Tepel M, Zidek W. Erythropoietin increases cytosolic free calcium concentration in vascular smooth muscle cells. Cardiovascular research1993;27(7): 1233-6.
46. Pirpiris M, Sudhir K, Yeung S, Jennings G, Whitworth J. Pressor responsiveness in corticosteroidinduced hypertension in humans. Hypertension1992;19(6):567-74.

47. Hawkey C, Langman M. Non-steroidal anti-inflammatory drugs: overall risks and management. Complementary roles for COX-2 inhibitors and proton pump inhibitors. Gut2003;52(4):600-8.

48. Warrington S, Padgham C, Lader M. The cardiovascular effects of antidepressants. Psychological medicine Monograph supplement1989;16(1):1-40.

49. Reckelhoff JF. Sex steroids, cardiovascular disease, and hypertension. Hypertension2005;45(2):170-4.

50. Hall GC, Brown MM, Mo J, MacRae KD. Triptans in migraine. Neurology2004;62(4):563-8.

51. Zhu X, Wu S, Dahut WL, Parikh CR. Risks of proteinuria and hypertension with bevacizumab, an antibody against vascular endothelial growth factor: systematic review and meta-analysis. American journal of kidney diseases2007;49(2):186-93.

52. Yeh ETH. Cardiotoxicity induced by chemotherapy and antibody therapy. Annu Rev Med2006;57:485-98.

53. First MR, Neylan JF, Rocher LL, Tejani A. Hypertension after renal transplantation. Journal of the American Society of Nephrology1994;4(8):S30-S6.

54. Baranowska-Kortylewicz J, Abe M, Pietras K, Kortylewicz ZP, Kurizaki T, Nearman J, et al. Effect of plateletderived growth factor receptor-Î̃ ${ }^{2}$ inhibition with STI571 on radioimmunotherapy. Cancer research 2005; 65(17): 7824.

55. Bhargava P. VEGF kinase inhibitors: how do they cause hypertension? American Journal of PhysiologyRegulatory, Integrative and Comparative Physiology 2009; 297(1):R1-R5.

56. RuthColeman. DRUGS THAT CAUSE CONGENITAL HEART DISEASE. 2010.

57. Lemire THSRJ. Catalog of Teratogenic Agents 2004.

58. Van Gelder MMHJ, Van Rooij IALM, Miller RK, Zielhuis GA, Roeleveld N. Teratogenic mechanisms of medical drugs. Human reproduction update;16(4):378-94.

59. Shyu KG. Serotonin 5-HT2B Receptor in Cardiac Fibroblast Contributes to Cardiac Hypertrophy. Circulation research2009;104(1):1-3.

60. McElhatton PR. The effects of benzodiazepine use during pregnancy and lactation. Reproductive toxicology (Elmsford, NY)1994;8(6):461. 